\title{
Business Network Formation Among 5G Providers
}

\author{
Mate Cserep*, Akos Recse*, Robert Szabo*, Laszlo Toka ${ }^{\dagger}$ \\ ${ }^{*}$ TrafficLab, Ericsson Research, Hungary \\ ${ }^{\dagger}$ MTA-BME Information Systems Research Group, MTA-BME Network Softwarization Research Group \\ Budapest University of Technology and Economics
}

\begin{abstract}
Virtualization ensures that in the approaching 5G era online services will be elastic and their deployments will be fast, fulfilling the demand of end-users rapidly and to a greater extent than what is feasible today. Telcos, cloud operators, and online application providers will join forces for delivering ICT services to customers globally. In order to support the mobility of customers, or the mere geographic span of an integrated enterprise application, the service deployments must span over many administrative domains and an assured quality of collaboration among various infrastructure and service providers is necessary. Therefore the vision of the $5 \mathrm{G}$ ecosystem is partly founded on the federation of these stakeholders in which they can seamlessly cooperate with the goal of creating the resource slices and the services within for a maximal geographic reach of customers. In this ecosystem, business aspects will greatly influence the technical capability and performance: we argue that the cooperative network of the actors will inherently determine availability and end-user prices of certain services. In this work we model the business relations of infrastructure providers as a variant of network formation games, and we derive conditions under which the current transit-peering structure of network providers remains intact.
\end{abstract}

\section{INTRODUCTION}

Keeping the operation of the autonomous networks manageable in today's Internet comes with the price of reducing the level of their interoperability to best effort. Therefore when one considers creating network services that span over multiple operators' domains, they can hardly assure Quality of Service $(\mathrm{QoS})$. The $5 \mathrm{G}$ vision however foresees online services to be more sophisticated than today's for which underlying infrastructure providers guarantee end-to-end QoS in network slices: low latency and high bandwidth. Furthermore, with the advent of virtualization both in compute and network technologies, faster service creation becomes possible and the reconfiguration of services can be more adaptive, resulting in a completely different service lifecycle management compared to what today's norm is. The concept of elastic resource slicing is the key enabler for this, and when multiple providers take part in creating a resource slice, similarly strict dedication to QoS assurance is required from all participants.

We argue that the locality of customers is one of the most important driving factors of the appearance of multi-provider resource slices. Network requirements of services inherently define the frame of eligible infrastructure for the underlying resource slice: the delay-sensitivity of certain virtual network functions (VNFs) determines the clouds or fogs in which they can be deployed close to the customers for delivering QoS. Another important factor is cost-efficiency: when QoS requisites allow, it can drive the need for multi-domain resource slice creation. Fortunately, Network Function Virtualization (NFV) makes it possible to create flexible services in form of Service Function Chains (SFC) of VNFs when the appropriate resource slice has been created beforehand.

Motivated by these factors, we foresee that $5 \mathrm{G}$ infrastructure providers will collaborate in federations. In order to be able to offer locality-sensitive services for their customers globally, they will use the compute and network resources of any of their partner providers. But before dedicated resource slices are provisioned spanning over multiple providers' administrative domains, business agreements have to be established between partners, e.g., on price and QoS guarantee of the infrastructure allocated to the resource slice. For the end-toend QoS assurance of a resource slice that many actors take part in, distributed negotiations and continuously maintained business relationships might be necessary among the actors. In this work we investigate how the network of business relations might evolve in the $5 \mathrm{G}$ era: will it follow the topology of transit and peering relations of the Internet today or will new business relations be established between adjacent or even remote providers similarly to ISPs' "peering" agreements?

We tackle the evolution of business relations as a network formation game. We make the case for two opposing forces. First, we account for a creation and maintenance cost of business relationships that cumulates as a provider establishes contracts with more and more actors. Second, we account for mediation prices by middleman actors if a provider initiates the creation of a resource slice with another provider with which it has no direct business link. We analyze the game from the perspective of profit-oriented $5 \mathrm{G}$ infrastructure providers in order to characterize this business trade-off. Our contribution in this paper is an analytical condition on these pricing factors for a stable business relationships topology. We derive analytical condition on the maximal middleman price for which no new business links are worth to be created. Moreover, in numerical results we show the essence of interplay between the prices, the demand for resource slices and the business topology.

The rest of the paper is organized as follows. In Sec. II we give an overview of related work in multi-provider pricing and network formation games. In Sec. III we introduce our business network model and the trade-off in details. In Sec. IV we formalize the model as a network formation game, and derive analytical results on equilibrium conditions. In Sec. V we turn to a numerical analysis, and show simulation results in a general setting. Finally we conclude our work in Sec. VI. 


\section{RELATED WORK}

To the best of our knowledge, there is no existing research that would tackle the pricing aspects of network or resource slices that are created by many actors. Nor we have found any work that investigated the specific cost factor due to maintaining business relations or the price of mediating business contracts to this end in a multi-provider setup.

A recent survey [1] reviews pricing models for resource management in cloud networking. Most of the collected works propose the application of dynamic pricing, as it increases seller's profit when two product characteristics co-exist: first, the product expires at a point in time, second, capacity is fixed and it is costly to be augmented. The term cloud networking is understood in a multi-administrative domain scenario in which network and data center domains interact with each other. Nevertheless, the exhaustive collection of related work presented in [1] does not include research results that tackle both multiple providers and various resource types to sell.

[2] introduced the network formation game that models the dynamic creation of networks by selfish node-agents without central design or coordination. In their model nodes pay for the links that they establish, and benefit from short paths to all destination nodes. The authors studied the Nash equilibria of the game, and derived results about the "price of anarchy", i.e., the relative cost of the lack of coordination. [3] studied a network formation game where links require the consent of both participants and are negotiated bilaterally, and compared these networks to those generated by the earlier model of [2] in which links are formed unilaterally. Their empirical analysis demonstrated that the average price of anarchy is better in the bilateral connection game than the unilateral game for small link costs but worse as links become more expensive. Another work that tackles bilaterally agreed contracts is presented in [4]: cost is incurred to a node from four sources: 1) routing traffic; 2) maintaining links to other nodes; 3) disconnection from destinations the node wishes to reach; and 4) payments made to other nodes. The authors study the game in perspective of a variation on the notion of pairwise stability. The difference compared to our work is that our model accounts for the "routing" term as income-generating, instead of making it a cost-increasing term. The book chapter [5] analyzes a number of various network formation games.

The authors of [6] investigated how the Internet ecosystem has been rapidly evolving from a multi-tier hierarchy built mostly with transit (customer-provider) links to a dense mesh formed with mostly peering links. The same authors published an agent-based network formation model for the Internet at the Autonomous System (AS) level in [7]: ASes act in a myopic and decentralized manner to optimize a cost-related fitness function, capturing key factors that affect the network formation dynamics, such as highly skewed traffic matrix, policy-based routing, geographic co-location constraints, and the costs of transit/peering agreements. In one of their more recent work [8], the same authors investigate why a large percentage of transit providers use an open peering strategy.

\section{Business Network Model}

We argue that the formation of future $5 \mathrm{G}$ business networks is highly dependent and thus will be initially based on the current Internet topology. Therefore we assume tiered structure following today's transit and peering relations as our initial network model to perform the evaluation on how the new $5 \mathrm{G}$ ecosystem may alter this topology. In this section we present the basic setup of our analysis in terms of costs of maintaining business relations, and the price of business middlemen.

\section{A. Graph Model of Business Relationships}

Figs. 1 and 2 show examples of our graph model of $5 \mathrm{G}$ infrastructure providers. In this model vertices represent i) network service providers (also offering compute infrastructure) and $i$ ) Service Access Points (SAPs), which are connection points for end-customers. Edges represent business relationships, which, in the initial phase can be either $i$ ) transit relations (represented by straight lines on the figure) or $i i$ ) peering relations (represented by dashed lines) between adjacent providers. The locality of customers and delay-sensitive services are among the most important driving factors of multiprovider ecosystems, therefore we depicted SAPs only at Tier3 providers as, in general, those provide access service to endusers. Note, however, that in our vision all network providers are potential $5 \mathrm{G}$ cloud providers.

We focus on such multi-domain services in which the endusers of the service are customers of provider A, while they actually use the service within the domain of provider B. In this case provider A buys a resource slice from provider B (and possibly from other providers interconnecting providers A and B). We model these business agreements as paths in the graph model connecting two nodes: the buyer of the service is the primary provider of the customer's Point of Presence, while the seller of the service is the provider in whose domain end-users will actually use the service. The delaysensitive service is therefore deployed at the seller provider's infrastructure close to the SAP of the end-user. Technical parameters of the specific service, such as compute resource demand, onboarding specific VNFs that constitute the service, network QoS to the SAP, etc. must be fulfilled and paid for by the buyer provider. In this multi-actor setup we analyze the formation of business relations: we do not tackle the pricing of the services or the resource slices, our focus is narrowed down to the pricing and costs of federation-related aspects.

\section{B. Cost of Business Relations and Price of Middlemen}

A service deployment in a resource slice that is mounted on third-party provider(s) infrastructure necessitates preestablished business agreements among the stakeholders. As described in Sec. I, we suppose that two "opposing forces" determine how these agreements are made: either by directly negotiating and billing, or through middlemen that provide mediation between the seller and buyer. In the latter case the base price of the resource slice is complemented by the mediation price of the interconnecting providers. The chain of mediating providers can be as long as the number of hops of 


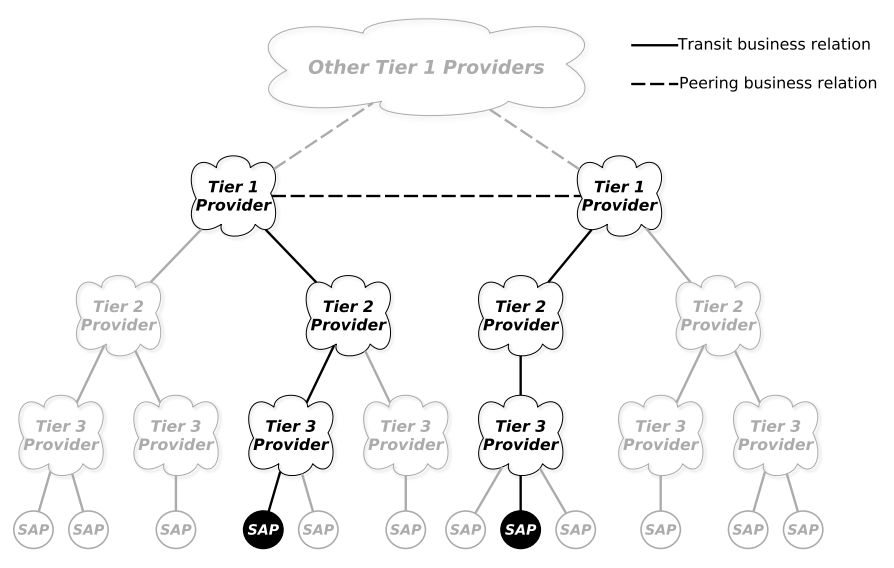

Fig. 1. The original service path between a selected pair of SAPs.

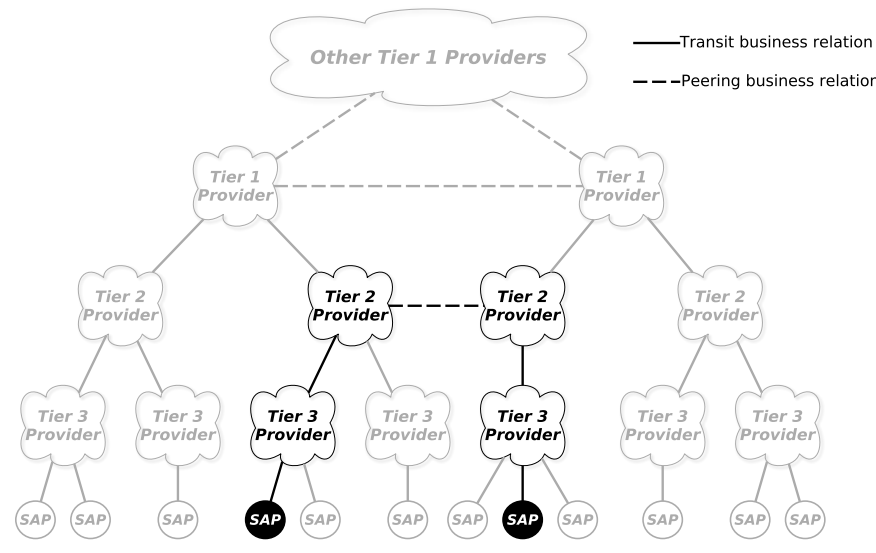

Fig. 2. A new possible service path in formed by establishing a new business connection between Tier-2 providers.

the path in our graph model. On the other hand, each additional direct business link between providers induces a cost at both parties: establishing and maintaining business contracts have their costs. The opposing effects is clear: while links increase the overall administrative costs, excluding middlemen from services deployed in remote resource slices is beneficial.

Fig. 1 depicts the service path between a selected pair of SAPs in an initial tiered graph model. The path goes through the transit relations towards the highest-tier providers in this example. However, a new service path can be formed by establishing business connections between any two of the involved intermediate providers - between Tier-2 providers in this example, as shown in Fig. 2. Observe, that the new business relation does not change the data plane path as the corresponding ISPs are not physically adjacent. Nevertheless, the mediated orchestration path is shortened as Tier-1 providers will not act as middlemen anymore. Again, they will potentially further provide connectivity services, which may be provisioned for the corresponding Tier-2 providers on a different timescale (e.g., for QoS traffic aggregates).

\section{Network Formation Game}

In this section we the define the variant of network formation game we use for modeling the previously introduced setup, then we derive a few important observations that characterize the equilibria of the game. Note that the main difference between our game variant and the existing ones listed in Sec. II is the cost function: first, the distance measure in our case is replaced by middleman costs, second, the cost of each player is reduced by the income generated by being a middleman.

\section{A. Game definition}

For tractability, we use the same notation as in [3]. We consider a network formation game in which players are the network service providers. $N$ denotes the player set $\{1,2, \ldots, n\}$. The strategy set of player $i$ is depicted by $S_{i}$ and it is the power set of $N \backslash i$, i.e., the collection of possible sets of other players to link with. We denote the link between nodes $i$ and $j$ by $s_{i j}$, therefore $S_{i}=\left\{\left(s_{i j}\right)_{j \neq i} \mid s_{i j} \in\{0,1\}\right\}$ and $\left|S_{i}\right|=2^{n-1}$. Each player $i$ plays a strategy $s_{i}$ out of its collection $S_{i}$, and the combination of the strategies of all players provide the outcome of the game. The resulted strategy profile is denoted by $s=\left(s_{1}, s_{2}, \ldots, s_{n}\right) \in S_{1} \times S_{2} \times \cdots \times S_{n}$. The outcome of this one-shot game is an undirected graph $G(s)=(N, E(s))$ in which a given edge is built if there is consent between the two nodes, i.e., $E(s)=\left\{(i, j): i \neq j, s_{i j}=1 \wedge s_{j i}=1\right\}$. That is both players $i$ and $j$ must agree to establish a link between each other in order for it to be created.

Cost function $c$ determines the player cost given the strategy profile out of the combination of strategy sets, i.e., $c: S_{1} \times S_{2} \times$ $\cdots \times S_{n} \rightarrow \mathbb{R}^{n}$. Similarly to related work, the cost incurred by player $i$ when all players adopt strategy $s$ is additive in the cost of the number of connections $\left|s_{i}\right|$ that provider $i$ establishes successfully with other providers, as well as in the sum of the middleman costs of doing business with all other providers. As a novel term, we also account for the income that is generated by acting as a middleman in businesses traversing provider $i$. In our game the cost function is defined as follows:

$$
\begin{aligned}
c_{i}(s) & =\alpha\left|s_{i}\right|+\sum_{j \in N \backslash i} \beta d_{(i, j)}(G(s)) M_{i, j} \\
& -\sum_{j \in N \backslash i} \sum_{k \in N \backslash i, j} \beta \mathcal{I}_{i \in p_{j, k}}(G(s)) M_{j, k} \quad \forall i \in N,
\end{aligned}
$$

where $\alpha$ and $\beta$ are the business peering cost and the middleman price introduced in Sec. III, respectively; $d_{(i, j)}(G(s))$ denotes the number of middlemen on the shortest-path between providers $i$ and $j$ in the business graph $G ; M_{i, j}$ depicts the extent of services bought by $i$ from $j$ through whatever path of middlemen providers this business is realized; and $\mathcal{I}_{i \in p_{j, k}}(G(s))$ indicates whether $i$ is on path $p_{j, k}$, i.e., the shortest path between $j$ and $k$. If no path exists between $i$ and $j$, then $d_{(i, j)}(G(s))=\infty$.

As in [3], this game model represents a network setting in which links are costly but good connectivity is desirable in order to minimize the number of middlemen to pay off. Also, the more links a provider has, the more likely it is going to act 
as a middleman, which generates income, hence lower total cost. Providers seek to minimize their costs defined in Eq. 1. If we assume that the cost of an additional business link $\alpha$, the middleman price $\beta$ and the service deployment request matrix $M$ are fixed, the game boils down to the following question: which new links are worth to be created in order to save cost.

\section{B. The effects of link creation}

For various types of equilibrium, stability conditions, efficient graphs, lower and upper bounds on the price of anarchy in classic network formation games, we refer the reader to [2], [3], [5]. However, note that the models therein are different from ours. The game variant closest to ours is presented in [4], but unlike to that model where a player's go-through traffic incurs cost, in our setup the more shortest paths traverse a node, the more income is generated to that provider. For their setup the authors proved that the stable outcome of the game is always a tree, as more transit paths and the link creation are not balanced by the value creation of lower distance to other nodes, once the graph is fully connected. In our game, on the other hand, the resulting graph $G(s)$ can be a tree intuitively only for high link creation $\operatorname{cost} \alpha$ : both lower distance to other nodes, and more traversing business paths decrease the cost, therefore if link creation is relatively cheap, it is beneficial.

As depicted in Sec. III, we assume an initial tiered topology of providers. The goal of the work presented in this section is to provide a sufficient condition under which there are no new links created by the providers. If this condition is satisfied, the initial tiered topology is therefore an equilibrium of our game.

Assumption 1. There are business links between providers originally, and these links organize nodes in a tiered topology, denoted by $G^{0}$, such as the one depicted in Fig. 1.

Let us number the tiers from top to bottom, $1,2, \ldots, t$, and let $t^{i}$ indicate the tier that provider $i$ belongs to. Let us denote by $C_{i}$ the set of providers that can be reached downwards in the tiered topology through provider $i$, i.e., $C_{i}=\{k \mid i \in$ $\left.p_{j, k} \forall j \mid t^{j}=1, t^{k}>t^{i}\right\}$, preferring peering links in tier-1 to peering links in lower tiers. Now we deduce the parametric cost saving when a new link is created.

Lemma 1. Given Assumption 1 holds, the highest cost reduction a new link between two nodes, denoted by $i$ and $j$, can achieve is

$2 \beta\left(t^{i}+t^{j}-2\right)\left|C_{i}\right|\left|C_{j}\right| \max \left(M_{k l \mid k \in C_{i}, l \in C_{j}}, M_{k l \mid l \in C_{i}, k \in C_{j}}\right)-2 \alpha$

Proof. Providers $i$ and $j$, belonging to tiers $t^{i}$ and $t^{j}$ respectively, would both make a cost reduction for their children in $C_{i}$ and $C_{j}$ by interconnecting themselves with a new link and thus lowering the second term of Eq. 1 of the children. At most $t^{i}-1+t^{j}-1$ middlemen in upper tiers are shortcut from cross paths between the two sets of children with the new link. This number might be lower if any peering links exist between parents of $i$ and $j$, or if they have the same tier-1 parent. Note that we assume full mesh among tier-1 providers in $G^{0}$. The cost allocated to middlemen is proportional with the extent of the business which is upper bounded by $\max \left(M_{k l \mid k \in C_{i}, l \in C_{j}}, M_{k l \mid l \in C_{i}, k \in C_{j}}\right)$. The number of business relationships is given by $\left|C_{i}\right|\left|C_{j}\right|$, hence the result starting from the following formula:

$$
\sum_{k \in C_{i}} \sum_{l \in C_{j}} \beta\left(t^{i}-1+t^{j}-1\right)\left(M_{k l}+M_{l k}\right)-2 \alpha .
$$

Hindered by the complexity in a general tiered topology setting, we make the following assumption on the number of children each node has, and of businesses leaf nodes make.

Assumption 2. $G^{0}$ contains a number of tier-1 nodes connected in full mesh, and a tree subgraph under each tier-1 node in which intermediary nodes have at least $k$ children, and all leaf nodes are at the same depth $t$. Furthermore, any pair of leaf nodes exchange $M$ amount of business; intermediary nodes do not act as service sellers or buyers.

Given the specific tiered topology of Assumption 2, we prove that the highest cost saving can be attained with new peering links in the topmost tier.

Lemma 2. Under Assumption 2, the higher tier the nodes belong to, the larger the cost saving that is attained if they create a new link.

Proof. Under Assumption 2 the size of $C_{i}$ and $C_{j}$ are lower bounded by the number of leaves of perfect k-ary trees: $\left|C_{i}\right| \geq$ $k^{t-t_{i}}$. The cost saving of two nodes $i$ and $j$ by creating a link is $2 \beta\left(t^{i}+t^{j}-2\right) k^{2 t-t^{i}-t^{j}} M-2 \alpha$. By expressing $x=t^{i}+t^{j}$, it is easy to see that this cost saving is higher when $\frac{x-2}{k^{x}}$ is larger. As $k \geq 2$ and $x \geq 3$, the maximum is attained if $x=3$, i.e., $t^{i}=1$ and $t^{j}=2$, or $x=4$, i.e., $t^{i}=2$ and $t^{j}=2$.

\section{Sufficient condition for status quo}

Let us assume dynamic pricing of top-tier providers in terms of mediation prices with the aim of excluding the economic reasons for new business links: as set out in the beginning of this section, we are interested in the pricing of middleman services when the goal of transit providers is to keep the status quo, i.e., eliminate the motivation of low-tier providers for creating business peering links. We derive the peering link exclusionary pricing for the topology that we assumed above.

Theorem 1. Under Assumption 2, if all providers keep their price below $\frac{\alpha}{k^{2 t-3} M}$, then topology $G^{0}$ is an equilibrium.

Proof. It is easy to see that the top-most tiers lose business if peerings are created underneath them. In such a topology $G^{0}$ that satisfies Assumption 2, the maximal middleman price $\beta$ for which no new links are worth to be created between any two providers is given by $2 \beta\left(t^{i}+t^{j}-2\right) k^{2 t-t^{i}-t^{j}} M-2 \alpha \leq 0$ from which the upper bound on $\beta$ is $\alpha \frac{k^{x}}{(x-2) k^{2 t} M}$ with $x=$ $t^{i}+t^{j}$, according to Lemma 2 . Result is yielded for $x=3$.

As a consequence of Theorem 1, the high-tier transit providers have an incentive to keep their middleman prices 
low. The fact that they want to preserve the status quo of transit-like business relations among providers has an overall positive effect on the ecosystem: the mediation price of establishing multi-domain services between remote (in the business sense) providers is upper bounded. This bound is dictated by the topology and the link creation cost.

\section{NUMERICAL ANALYSIS}

In order to perform further analysis on our theoretical results we constructed a simulation environment.

\section{A. Simulation setting}

Since we examine the evolution of the business agreements that define today's Internet, a topology following its scheme was required as an initial state: the input topology of the simulations is the one introduced in Sec. III. We created 3 tiers and an additional layer of SAPs below tier-3. To be aligned with the requirement on minimum width applied in Sec. IV, we picked a random number of children for each node in the tree from a uniform distribution between 5 and 10. Thus the simulation consisted of approximately 300 providers.

Instead of a constant value for all SAP-to-SAP demand of service, we assumed heterogeneous demand, e.g., regional hot spots that customers from other regions are more likely to pick for deploying services in resource slices. Accordingly, we constructed the business matrix by using Gaussian functions that peek at a randomly selected providers.

The link creation and middleman costs, $\alpha$ and $\beta$ as described in Sec. IV, are parameters that can be analyzed relative to each other. We decided to fix the $\alpha$ parameter to 1 and perform simulations with several $\beta$ values.

\section{B. Iterative simulation}

Our simulation is divided into rounds with each containing a complete analysis: all providers try to determine their optimal set of business connections. If there are no further changes from one round to the other, the simulation has converged and the status represents an equilibrium state of the topology.

Within a simulation round, providers check for all other providers if a new connection is worth to establish based on the cost function defined in Sec. IV. If the selected partner also decides to create the connection, a new business relation (an edge in the graph) will be added to the topology. Existing business connections are also examined in each simulation round whether their maintenance is still beneficial to both parties or not - disadvantageous connections are dropped.

\section{Simulation results}

After running hundreds of simulations with several initial setups we compare final topologies from the perspective of various metrics that we depict in the following figures.

In Fig. 3 we can observe how the alteration of middleman prices, $\beta$ 's value, transforms the structure of the initially tiered topology. The figure shows the number of new connections, separated by tiers, comparable for various middleman prices, shown on a logarithmic $x$ axis. In the left-end middleman cost

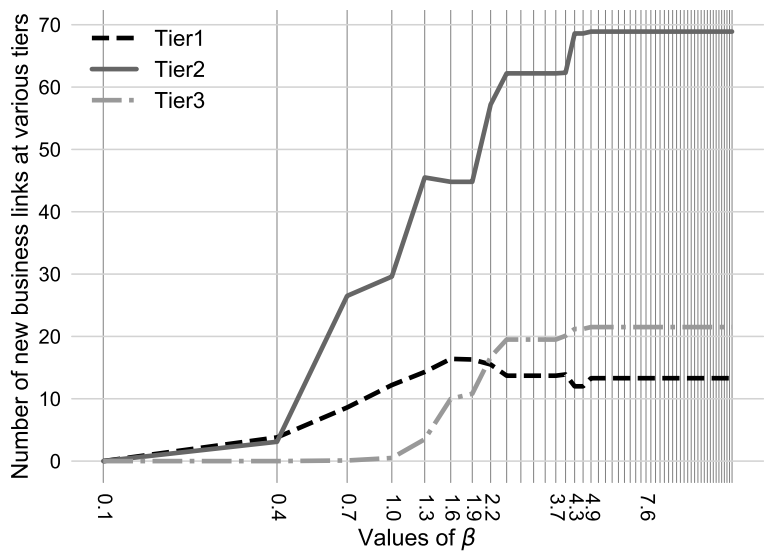

Fig. 3. Number of new business connections, by tiers, for various $\beta$ values.

is low enough to strongly limit the establishment of new business peerings. As $\beta$ grows compared to the maintenance cost of a business relation, i.e., $\alpha$, every provider starts to shorten their business paths in order to avoid expensive middlemen in upper tiers. As both parties must agree to form a business peering and the traffic demand between tier- 3 providers is rarely large enough mutually, most business connections involve at least one upper-tier provider. Simultaneously, the remaining aggregated "transit" business towards the top-tier providers is reduced, therefore some of the initially established business relationships for tier-1 providers are dropped in the later iterations of the simulation. Approximately $\beta=4.3$ is the point where providers route most business through peerings.

Intuitively the more popular a tier-3 provider's locality is, the shorter the paths others would like to reach it through. Based on Fig. 3 we picked 4 interesting cases: $i$ ) $\beta=0.4$ limited number of business peerings in all tiers, ii) $\beta=1.3$ - connections of tier-1 providers is around its peak, iii) $\beta=2.2$ - tier-1 providers drop some connections while business peering is on the rise in tier- 3 and $i v) \beta=7.6$ a convergence in the number of new business connections has been reached. Fig. 4 depicts the total amount of service sold by

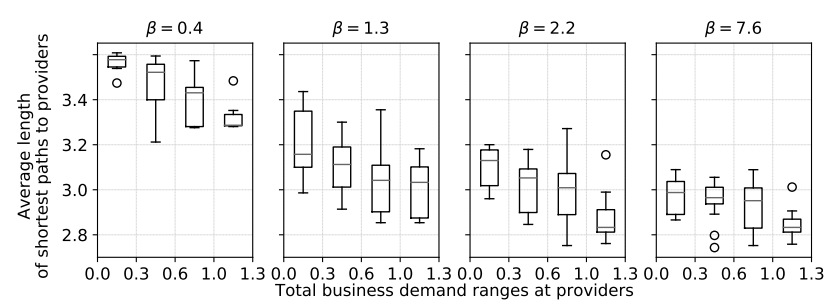

Fig. 4. Average length of paths to providers by the amount of service sold.

the providers (sum of the corresponding column in the business matrix) divided into four value ranges, while on the $y$ axis the average path length to the providers belonging to the particular range is visualized. For all four $\beta$ values a decreasing trend can be noticed on all sub-figures. A global decrease of lengths 
is also observable as the value of $\beta$ is increased, making the establishment of new business connections more beneficial.

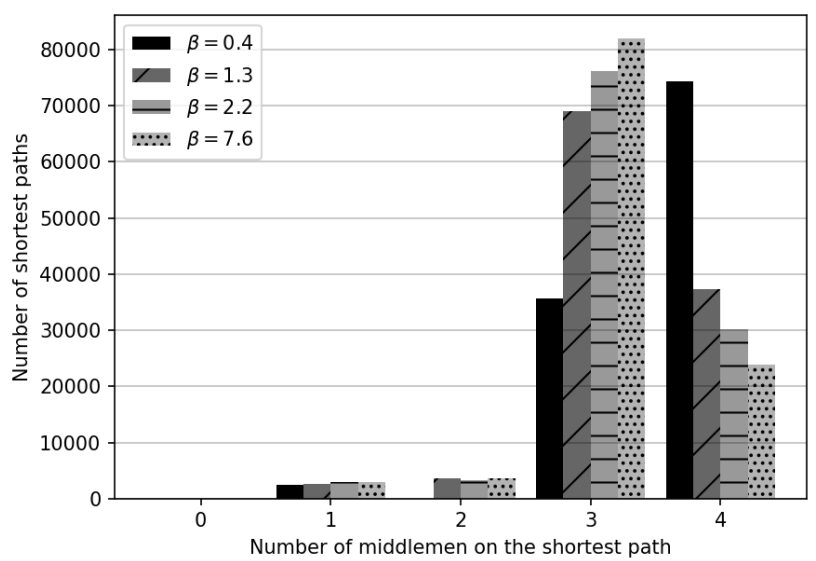

Fig. 5. Number of shortest paths by the number of their middlemen

The length distribution of business paths in the topology is presented in Fig. 5: it shows the number of middlemen along the shortest business paths in the stable topologies created for different values of $\beta$. The maximum value this metric can obtain is 4 , as in this case there are no shortcuts in the tree: the shortest path is through tier-1 providers. One can realize how routes shorten as $\beta$ grows, while most connections are formed between upper-tier providers, hence many 3-hop and 4-hop paths. The number of paths with 0 middlemen is close to 0 in all scenarios, verifying that peerings were rarely formed between tier-3 providers, as concluded in Fig. 3 .

\section{DISCUSSION}

Cloud computing has proliferated in the recent past as the de facto standard thanks to offering cheaper and easier solutions for IT services. Distributed computing seems to be the next major step forward to maximize service performance in a costefficient way. Market fragmentation, however, will make it necessary for Telco providers to cooperate (and to ompete) in their distributed cloud offerings. What kind of business relations will emerge among Telcos is an open question. There are new alliances formed, e.g., ngena ${ }^{1}$, by key stakeholders to pioneer solutions. The EU H2020 5G Exchange project ${ }^{2}$ aims at establishing the technical enablers for an open coopetitive (competitive cooperation) ecosystem for multi-provider network service management. In the frame of our actor-role and business case analysis we set the goal to systematically analyze possible emerging business structures for distributed cloud offerings. Given, however, the current multi-tier ISP hierarchy for global connectivity services, we started our analysis by looking into why, when and how the current ISP structure may transform into new business relationships.

\footnotetext{
${ }^{1}$ http://www.ngena.net/

${ }^{2}$ http://www.5gex.eu/
}

Starting from the tiered ISP hierarchy, we defined a network formation game in which players are the ISPs themselves; link creation represented business relationships with a maintenance cost; operational costs and incomes were calculated based on paid transit costs and incomes from being a middleman (offering transit services). Connectivity and distributed cloud services were routed along the traditional and the enriched ISP structures respectively, since new business relationships do not necessarily imply physical adjacency of the corresponding providers. With further assumptions (see Sec. IV-B) we derived a formal upper bound on the middleman price as a function of peering costs and topology attributes, i.e., if ISPs would like to preserve the current status quo with respect to their transit relationships, then they have to keep their middleman (mediation) prices low. The analytical results' dependency on the topology promised different price tags for each tier.

In our numerical analysis we simulated games with different middleman costs for various initial ISP topologies. In a 3-tier hierarchy our results revealed that with increasing middleman costs the number of business connections to be established also rises until when a convergence state is reached where no new peerings are affordable due to the lack of traffic demand. Results also showed that the mutual desire for peering among the lower tier-3 level providers rarely occurs, instead the intermediate tier- 2 providers manage to attract most of the new business due to their capability of aggregating the transit demands of the sub-ordinate ISP levels. Even though our simulations were run with global peering and middleman price values, we believe that individual player strategies could exploit the multiple thresholds given by the model.

In our future work we plan to look into a dynamic simulation to analyze the maximum achievable revenue for a player in the view of its individual middleman price strategy. We are also interested in how impartial information will affect the outcome of the game, like unknown and unbalanced graphs.

\section{ACKNOWLEDGEMENT}

This research was supported by H2020-ICT-2014 project 5GEx (grant agreement no. 671636), which is partially funded by the European Commission. Project no. PD 121201 has been implemented with the support provided from the National Research, Development and Innovation Fund of Hungary, financed under the PD_16 funding scheme.

\section{REFERENCES}

[1] N. C. Luong et al., "Resource management in cloud networking using economic analysis and pricing models: A survey," IEEE Communications Surveys Tutorials, vol. 19, no. 2, pp. 954-1001, 2017.

[2] A. Fabrikant et al., "On a network creation game," in ACM PODC, 2003.

[3] J. Corbo et al., "The price of selfish behavior in bilateral network formation," in ACM PODC, 2005.

[4] E. Arcaute et al., "Network formation: Bilateral contracting and myopic dynamics," IEEE Transactions on Automatic Control, vol. 54, no. 8, pp. 1765-1778, 2009.

[5] E. Tardos et al., Algorithmic Game Theory, 2007, no. Chapter 19: Network formation games and the potential function method.

[6] A. Dhamdhere et al., "The internet is flat: Modeling the transition from a transit hierarchy to a peering mesh," in ACM CoNEXT, 2010.

[7] A. Lodhi et al., "GENESIS: An agent-based model of interdomain network formation, traffic flow and economics," in IEEE INFOCOM, 2012.

[8] — "Open peering by internet transit providers: Peer preference or peer pressure?" in IEEE INFOCOM, 2014 